\title{
On the thermodynamic origin of the uncertainty principle
}

\author{
Jay R. Yablon ${ }^{1}$ \\ ${ }^{1}$ Einstein Centre for Local-Realistic Physics; yablon@alum.mit.edu
}

\begin{abstract}
The spatial resolution measurement limitation of the position-momentum uncertainty principle is shown to mathematically originate from the Bekenstein entropy bound and the associated second law of thermodynamics, as a special case in which the average energy of a statistical thermodynamic distribution of energies is specialized to a fixed, definite probe energy. This is used in combination with the Wein displacement law to predict an ultraviolet cutoff for Planck blackbody radiation at about $1 / 8$ of the Wein peak. A new UV photon counting experiment is proposed to test for this. A physical understanding of these results may be provided by a UVcomplete, intelligible theory of general relativistic quantum mechanics in which the observation of a blackbody spectrum is simply a remote observation of Hawking radiation emitted from black hole fluctuations in the gravitational vacuum.
\end{abstract}

Keywords: entropy; statistical thermodynamics; uncertainty principle; Bekenstein bound; Hawking radiation; blackbody radiation; Wein displacement law; black holes; second law of thermodynamics; general relativity and quantum mechanics unification

\section{Introduction}

The position-momentum uncertainty principle first formulated by Heisenberg [1] and implied by Born's commutation relations [2] has long been part of the canonical foundation of quantum mechanics. We demonstrate how the spatial resolution measurement limitation of the uncertainty principle can be mathematically derived as a special case of a deeper relation between temperature and length arising from the Bekenstein entropy bound and associated second law of thermodynamics. When combined with the Wein displacement law, this predicts an ultraviolet cutoff at about $1 / 8$ of the Wein peak for black holes and ordinary blackbodies alike. Because Planck's law [3] predicts, statistically speaking, that for every 245.61 billion photons emitted near the Wein peak there will be only one photon emitted near $1 / 8$ of that peak, a new experiment to detect this UV cutoff is proposed to see whether in fact, after cataloguing trillions of photon emissions, there are in fact no emissions at all below about $1 / 8$ of the Wein peak, as predicted here. Because Planck-scale black holes are conjectured to permeate the gravitational vacuum first described by Wheeler [4], [5], this appears, with UV-completeness, to ground canonical quantum mechanics in the general theory of relativity which ab initio predicts black holes and their event horizons beyond which - as with the uncertainty principle - external observations cannot penetrate.

\section{The Bekenstein bound, Bekenstein-Hawking black hole entropy, and Hawking black hole radiation}

In 1981 Bekenstein [6] found that any system enclosing a total energy $E$ within a sphere of radius $R$ has an entropy $S$ with an upper limit rooted in the second law of thermodynamics:

$$
S \leq \frac{2 \pi k R E}{\hbar c} .
$$

Black holes are entropically saturated, so their entropies are equal to the maximum of this Bekenstein bound. Thus, for the special case of a Schwarzschild black hole with energy $E=E_{B H}=M c^{2}$ and 
Schwarzschild radius $R=r_{S}=2 G M / c^{2}$, we may use the Planck energy $E_{P}=\sqrt{\hbar c^{5} / G}$, the reduced Planck length $\lambda_{P}=\sqrt{\hbar G / c^{3}}$ and an event horizon surface area $A_{S}=4 \pi r_{S}{ }^{2}$, and apply the equality in (1.1), to obtain the Bekenstein-Hawking (BH) entropy:

$$
S_{B H}=\frac{2 \pi k R E}{\hbar c}=4 \pi k \frac{G}{\hbar c^{5}} E^{2}=4 \pi k \frac{E^{2}}{E_{P}{ }^{2}}=4 \pi k \frac{M^{2}}{M_{P}{ }^{2}}=\pi k \frac{c^{3}}{\hbar G} r_{S}^{2}=\pi k \frac{r_{S}{ }^{2}}{\hbar_{P}{ }^{2}}=k \frac{A_{S}}{4 \lambda_{P}{ }^{2}} .
$$

This reproduces what Bekenstein originally discovered in 1972 [7] and later generalized in [6] as shown in (1.1), which is that black hole entropy varies linearly with surface area not volume. ' $t$ Hooft [8] subsequently characterized this as a holographic principle.

If we differentiate the mass relation in (1.2) using $d$ for state and $\delta$ for non-state functions as is customary, we obtain $d S_{B H} / \delta M=8 \pi k M / M_{P}{ }^{2}$. Simultaneously, for pure heat transfer, with a mass change arising only from energy not matter entering or exiting the system, e.g., via capture or emission of massless photons, the second law of thermodynamics may be stated as $d S=c^{2} \delta M / T$. Rewriting this as $d S / \delta M=c^{2} / T$ then combining the two entropy relations, we obtain $8 \pi k M / M_{P}^{2}=c^{2} / T$, rewritten as:

$$
k T=\frac{c^{2} M_{P}^{2}}{8 \pi M}=\frac{\hbar c^{3}}{8 \pi G M}=\frac{\hbar c}{4 \pi r_{S}} .
$$

First discovered by Hawking in 1974 [9], this gives the characteristic temperature $T$ of the black hole Hawking radiation blackbody spectrum viewed by a distant observer (formally, at spatial infinity) following the gravitational redshift of photon energies, see e.g. eq. (14.3.8) of [10].

We also merge (1.2) and (1.3), again using $\lambda_{P}=\sqrt{\hbar G / c^{3}}$ and $r_{S}=2 G M / c^{2}$, to find that:

$$
S_{B H} T=\frac{\pi k r_{S}^{2}}{\hbar_{P}^{2}} \frac{\hbar c}{4 \pi k r_{S}}=\frac{\hbar c}{4 \hbar_{P}^{2}} r_{S}=\frac{\hbar c^{4}}{4 \hbar G} \frac{2 G M}{c^{2}}=\frac{M c^{2}}{2}=\frac{E_{B H}}{2}
$$

The factor of $1 / 2$ in the above originates mathematically from the relation $x d x=\frac{1}{2} d\left(x^{2}\right)$ for any variable $x$, because the entropy in (1.2) is proportional to the square of the mass. Specifically, in natural $\hbar=c=G=k=1$ units, an entropy accruing according to $d S_{B H}=8 \pi M d M$ which was used with $d S=\delta M / T$ to go from (1.2) to (1.3), integrates up to $S_{B H}=4 \pi M^{2}$ in (1.2). So in this way, the constant $1 / 2$ in (1.4) encodes the integral accrual of entropy and energy into a black hole.

\section{Derivation of the uncertainty principle spatial resolution measurement limitation from the Bekenstein bound and the associated second law of thermodynamics}

Now consider a general system which may or may not be a black hole. Because black holes are entropically-saturated, define a first parameter $0 \leq \alpha \equiv S / S_{B H} \leq 1$ denoting the fractional relation of $S$ to $S_{B H}$. For a black hole $\alpha=1$, otherwise $\alpha<1$. Then use this to rewrite (1.1) as $S=\alpha 2 \pi k R E / \hbar c$. Next, consider $\frac{1}{2} E_{B H}=S_{B H} T$ from (1.4). Writing the second law of thermodynamics as $\delta E \leq T d S$ with $E$ instead of $Q$, we see the change in entropy will either outpace the change in energy, or both will vary at the same pace. So the generalization of (1.4) from black holes to any system is $\frac{1}{2} E \leq S T$. Accordingly, define a second parameter $0 \leq \beta \leq 1$ where $\beta=1$ for a black hole, then write the energy generally as $\frac{1}{2} E \equiv \beta S T$. Again, the $1 / 2$ encodes entropy and energy accrual into a black hole. Then multiply $S=\alpha 2 \pi k R E / \hbar c$ through by $\beta T$ and use all the foregoing to rewrite Bekenstein's (1.1) as:

$$
\frac{1}{2} E=\beta S T=\alpha \beta \frac{2 \pi R E}{\hbar c} k T .
$$


Dividing out $E$ from the first and last terms, and given that both $\alpha$ and $\beta$ are less than or equal to 1 , this is easily restructured into:

$$
\frac{\hbar c}{2 \alpha \beta}=2 \pi R k T \geq \frac{\hbar c}{2} .
$$

If we reintroduce the definitions of $\alpha$ and $\beta$ into the equality above we recover $S_{B H}=2 \pi R E k / \hbar c$ which is the equality (1.2) in the Bekenstein bound (1.1). Therefore, given that $\alpha$ and $\beta$ both have an upper bound of 1 , the inequality $2 \pi R k T \geq \hbar c / 2$ in the above is an entirely equivalent way of writing the generalized Bekenstein bound (1.1) in view of Hawking's (1.3). The entropic accrual constant $1 / 2$ is now multiplies $\hbar c$, which in particle physics has dimensions of interaction strength. The equality $2 \pi R k T=\frac{1}{2} \hbar c$ in (2.2) applies to a black hole for which $\alpha=\beta=1$.

Now consider a system in thermal equilibrium for which each degree of freedom has average equipartition energy $\langle E\rangle=\frac{1}{2} k T$. Use this in (2.2) with system diameter $D=2 R$, yielding $2 \pi D\langle E\rangle \geq \frac{1}{2} \hbar c$. Then define wavelength $\lambda$ and reduced $\lambda$ by $D \equiv \lambda \equiv \lambda / 2 \pi$, so (2.2) becomes:

$$
\langle E\rangle \lambda \geq \frac{1}{2} \hbar c
$$

For a system with a large number $N$ of independent degrees of freedom, and labeling the energy of each degree of freedom by $E_{n}$, this average is $\langle E\rangle=\sum_{n=1}^{N} E_{n} / N$.

Then, instead of applying a temperature to the system to impart a collection of energies $E_{n}$ which average out to $\langle E\rangle$, apply a fixed, definite energy $\mu$ to probe the system. This is precisely what is done in particle physics collision experiments to probe molecules and atoms and nuclei and nucleons, see e.g. section 1.4 of [11] and the conversion constant $\hbar c=.1973269804 \mathrm{GeV} \mathrm{fm}$ in the $2019 \mathrm{PDG}$ data [12]. We then obtain a special case of (2.3) in which the average energy is specialized to a fixed, definite energy $\mu \equiv\langle E\rangle$ with each $E_{n}=\langle E\rangle$, thus a standard deviation $\Delta\left(E_{n}\right)=0$. With this, (2.3) specializes to $\mu \lambda \geq \frac{1}{2} \hbar c$. But because we have restricted the statistical distribution of $E_{n}$ to a single definite energy, we may likewise use $\Delta r \equiv \lambda$, reflective of wave-particle duality, to define a fixed length deviation. Consequently, specializing (2.3) to probes of fixed definite energy and fixed length deviation yields:

$$
\mu \Delta r \geq \frac{1}{2} \hbar c .
$$

This, of course, is the widely-recognized spatial resolution measurement limitation rooted in the uncertainty principle $\Delta p_{i} \Delta x_{j} \geq \frac{1}{2} \delta_{i j} \hbar$, which is in turn rooted in the canonical commutator $i\left[\hat{p}_{i}, \hat{x}_{j}\right]=\hbar \delta_{i j}$ of quantum mechanics via the Robertson uncertainty principle [13]. It is remarkable that (2.4) which plays a central role in particle physics and quantum mechanics generally, is merely a special case of the more general relations (2.2) and (2.3) rooted in statistical thermodynamics. Specifically, it is striking that this well-established uncertainty-based relation (2.4) is just a special case of the Bekenstein bound (1.1) in view of Hawking's (1.3) in which the statistically-distributed $E_{n}$ all become equal to the average energy $\langle E\rangle$ of that distribution.

\section{Prediction of an ultraviolet cutoff in Hawking and Planck blackbody radiation for wavelengths below about one-eighth of the Wein displacement peak}

The Wein displacement law for the peak wavelength of a Planck blackbody distribution is:

$$
\lambda_{\text {peak }} T=b=\frac{2 \pi \hbar c}{a k}=2.89771955 \times 10^{-3} \mathrm{~m} \mathrm{~K} .
$$

Listed in [12], this is obtained by the well-known calculation of $d B_{\lambda}(\lambda, T) / d \lambda=0$ from Planck's blackbody law, during which one obtains $a e^{a} /\left(e^{a}-1\right)-5=0$ thus a constant $a=4.965114232$. 
As already reviewed, (2.2) is merely a restatement of the generalized Bekenstein bound (1.1) in view of Hawking's (1.3). So, setting $2 R=D=\lambda$ in (2.2) then rewriting this as $\lambda T \geq \hbar c / 2 \pi k$, then taking the ratio of this over (3.1) and reducing, we obtain:

$$
\frac{\hbar}{\lambda_{\text {peak }}} \geq \frac{a}{4 \pi^{2}}=0.125767813=\frac{1}{7.951159986} \cong \frac{1}{8} \text {. }
$$

What we discover in (3.2), is that combining the Bekenstein bound in the form of (2.2) with Wein's (3.1) leads to a new prediction that there is an ultraviolet cutoff $\lambda_{\text {cutoff }}$ near $1 / 8$ of the Wein peak wavelength in all Planck radiation emitted from blackbodies and black holes alike, below which no shorter-wavelength photons are emitted at all. It is important to state that this UV cutoff predicted in (3.2) is not speculative. It is an inexorable mathematical consequence of combining the Bekenstein bound (1.1) represented in the form of (2.2) with Wein's displacement law (3.1). Mathematically, the inequality in the Bekenstein bound, which derives from the inequality in the second law of thermodynamics for irreversible systems, is also the inequality in the uncertainty principle and the inequality in the UV cutoff (3.2).

To gain a physical sense for why this occurs, first write (2.2) as $k T \geq \hbar c / 4 \pi R$. In the special case where the system radius $R=r_{S}$ is a black hole radius, the equality precisely recovers Hawking's (1.3). Because $\bar{\lambda}=d_{S}=2 r_{S}$ is the Schwarzschild diameter of this black hole, photon "particles" are trapped in a black hole "box" and cannot escape when their wavelengths $\lambda \leq d_{S}$ are less than or equal to the diameter of the black hole, which likewise connects ordinary spatial lengths with photon radiation wavelengths. This very same approach is used by Susskind in his lecture [14] to derive Bekenstein's black hole entropy relation (1.2).

But (3.2) makes this even more general: It tells us that a generalized diameter $D=2 R$ from the Bekenstein bound (1.1) for any system emitting blackbody radiation similarly acts as the diameter of a "box," trapping all photon emissions with wavelengths below about $1 / 8$ of the Wein peak. The inequality in (3.2) which signifies this ultraviolet cutoff is thus seen to be rooted in the inequality of the Bekenstein bound (1.1), and so in turn, in the second law of thermodynamics. Consequently, at bottom, the second law of thermodynamics forms the foundation of the ultraviolet cutoff in (3.2), and also of the uncertainty principle relation (2.4). Eq. (3.2) is simply how the uncertainty principle measurement limitation manifests more generally in thermodynamic blackbody systems. Possible theoretical implications of this will be reviewed in section 5. But first, we propose a new experiment by which this predicted UV cutoff might be detected.

\section{Proposed photon counting experiment for detecting the predicted UV cutoff in Planck blackbody radiation}

An experiment to directly detect the ultraviolet cutoff predicted at $\lambda_{\text {cutoff }} \cong \frac{1}{8} \lambda_{\text {peak }}$ by (3.2) for blackbody radiation proceeds as follows: Simply heat an ordinary blackbody, then detect and count photons both near $\lambda_{\text {peak }}$ and just below $\frac{1}{8} \lambda_{\text {peak }}$, in very large numbers, to see if there really is the complete ultraviolet cutoff predicted by (3.2). As a predicate, we need to calculate how many photons are predicted by Planck's law in the vicinity of $\frac{1}{8} \lambda_{\text {peak }}$ - which we expect will be a very small number - relative to the number of photons predicted right near $\lambda_{\text {peak }}$. The experiment is then to detect whether this very small number really is observed, or whether, as predicted in (3.2), no photons at all are observed.

A simple way to calculate this is to reparameterize Planck's law along dimensionless $x$ and $y$ axes re-scaled so the Wein peak is always at the point $(x, y)=(1,1)$ regardless of temperature, then find the height of $y$ at $x \sim 1 / 8$. This provides the relative energy output which Planck's law predicts near the UV cutoff of (3.2). Then, because the energy of a single photon varies inversely with its wavelength, we must divide the energy result through by a factor of $\sim 8$ to obtain photon numbers rather than energies. 
So, start with Planck's law [3] for the power density of a blackbody with temperature $T$ in terms of dependency on wavelength $\lambda$ :

$$
B_{\lambda}(\lambda, T)=\frac{4 \pi \hbar c^{2}}{\lambda^{5}} \frac{1}{\exp (2 \pi \hbar c / \lambda k T)-1} .
$$

Next, define a dimensionless ratio $x \equiv \lambda / \lambda_{\text {peak }}$ to label an $x$-axis, then use this together with Wein's (3.1) to reparametrize (4.1) by:

$$
B_{\lambda}\left(x, \lambda_{\text {peak }}\right)=\frac{4 \pi \hbar c^{2}}{x^{5} \lambda_{\text {peak }}^{5}} \frac{1}{\exp (a / x)-1} .
$$

$B_{\lambda}$ at the horizontal axis coordinate where $x=\lambda / \lambda_{\text {peak }}=1$ in (4.2) is easily found to be:

$$
B_{\lambda}\left(x=1, \lambda_{\text {peak }}\right)=\frac{4 \pi \hbar c^{2}}{\lambda_{\text {peak }} 5} \frac{1}{\exp (a)-1} .
$$

Finally, take the ratio of (4.2) to (4.3), then define the desired dimensionless function:

$$
y(x) \equiv \frac{B_{\lambda}\left(x, \lambda_{\text {peak }}\right)}{B_{\lambda}\left(x=1, \lambda_{\text {peak }}\right)}=\frac{1}{x^{5}} \frac{\exp (a)-1}{\exp (a / x)-1} .
$$

It will be appreciated that the peak wavelength $\lambda=\lambda_{\text {peak }}$ i.e. $x=1$ is always situated at the fixed coordinate point $(x, y)=(1,1)$ and the shape of $y(x)$ is invariant with regard to temperature.

Now, insert $x=x_{\text {cutoff }}=\lambda_{\text {cutoff }} / \lambda_{\text {peak }}=0.125767813$ from (3.2) into (4.4) to obtain:

$$
\begin{aligned}
& y\left(x_{\text {cutoff }}=0.125767813\right) \equiv \frac{B_{\lambda}\left(x_{\text {cutoff }}, \lambda_{\text {peak }}\right)}{B_{\lambda}\left(x=1, \lambda_{\text {peak }}\right)}=\frac{1}{x_{\text {cutoff }}^{5}} \frac{\exp (a)-1}{\exp \left(a / x_{\text {cutoff }}\right)-1} . \\
& =3.237237234 \times 10^{-11}=1 / 3.089053806 \times 10^{10}
\end{aligned}
$$

This says that the photon energy emitted at $x=x_{\text {peak }}=1$ is predicted by Plank's law to be stronger than that at $x=x_{\text {cutoff }}=0.125767813 \cong \frac{1}{8}$ by a factor of $3.089053806 \times 10^{10}$, or about 30.89 billion.

But, (4.5) is a relative energy, not a photon count. Each photon emitted near $x_{\text {cutoff }} \cong \frac{1}{8}$ has a shorter wavelength $\lambda$ and higher frequency $v=c / \lambda$ thus higher energy $E_{\gamma}=2 \pi \hbar v$ than each near $x_{\text {peak }}=1$ by a factor of about $1 / x_{\text {cutoff }} \cong 8$. So, to turn (4.5) into a ratio of photon numbers $n_{\gamma}$ rather than energy emission we must divide through by this factor of just under 8 . This is achieved by multiplying (4.5) through by $x_{\text {cutoff }}$. Accordingly, for numbers of photons, we obtain:

$$
\begin{aligned}
& x_{\text {cutoff }} \cdot y\left(x_{\text {cutoff }}=0.125767813\right) \equiv \frac{n_{\lambda}\left(x_{\text {cutoff }}, \lambda_{\text {peak }}\right)}{n_{\lambda}\left(x=1, \lambda_{\text {peak }}\right)}=\frac{1}{x_{\text {cutoff }} 4} \frac{\exp (a)-1}{\exp \left(a / x_{\text {cutoff }}\right)-1} . \\
& =4.071402462 \times 10^{-12}=1 / 2.456156102 \times 10^{11}
\end{aligned}
$$

Consequently, Planck's law predicts that for every 245.61 billion photons observed near $\lambda_{\text {peak }}$ there will be only one photon observed near $\frac{1}{8} \lambda_{\text {peak }}$. This is an extremely rapid ultraviolet diminution already built into Planck's law. But although exceptionally small, this is not zero. So, the specifics of the proposed experiment are as follows:

Heat an ordinary blackbody cavity to thermal equilibrium at any convenient temperature, then count photons both near $\lambda_{\text {peak }}$ and just below $\frac{1}{8} \lambda_{\text {peak }}$, in very large numbers, to see if there really is the complete ultraviolet cutoff predicted by (3.2), or whether observations show one photon on average near $1 / 8$ of the Wein peak for every 245.61 billion near the peak. To contradict the prediction (3.2), one needs to verifiably detect only a single photon with $\lambda<\frac{1}{8} \lambda_{\text {peak }}$ against about 245.61 billion near $\lambda_{\text {peak }}$ predicted by Planck's law. Conversely, confirming this cutoff would require observing 
and counting thousands of trillions of photons before 5-sigma confidence could be established that this cutoff truly exists in nature. The equipment needed for this is reasonably uncomplicated and inexpensive: a heated blackbody and a photon detector tuned to both $\lambda_{\text {peak }}$ and to wavelengths under $\sim \frac{1}{8} \lambda_{\text {peak }}$, coupled with a device that can count the detected photons into the quadrillions.

\section{Outlines of a UV-complete general relativistic theory of quantum mechanics}

As noted following (3.2), using "particle in a box" analysis, it is straightforward to envision how a black hole would trap wavelengths shorter than or equal to its diameter, producing the UV cutoff (3.2) for its Hawking radiation spectrum. Again, this is the approach Susskind uses in [14] to derive Bekenstein's (1.2). It is less straightforward to envision this for a generalized blackbody. Thus, we now examine why the gravitational vacuum may well provide the best explanation for why the UV cutoff (3.2) applies not only to black holes, but to blackbodies in general.

If one was only aware of the uncertainty principle measurement limitation (2.4) and not its thermodynamic generalization (2.2) based on the Bekenstein bound (1.1), then one could conclude that for a blackbody generally, the lower wavelength boundary based on uncertainty would be $E_{n} \lambda_{n} \geq \frac{1}{2} \hbar c$ rather than $\langle E\rangle \lambda \geq \frac{1}{2} \hbar c$ in (2.3), whereby a set of lower wavelength boundaries $\lambda_{n} \geq \frac{1}{2} \hbar c / E_{n}$ would be connected to each individual energy $E_{n}$ in the manner of the uncertainty limitation (2.4). In this event, given a statistical thermodynamic distribution including some very large $E_{n}$ within a blackbody thus very short $\lambda_{n}$ on the ultraviolet end of the distribution, there would be no cutoff at any particular short wavelength. But (2.2) and (2.3) are based on and are indeed a restatement of the Bekenstein bound of statistical thermodynamics. And these make clear that there is a single lower wavelength bound arising from the average energy of the degrees of freedom in the system that is, from the temperature of the system - rather than there being differing lower bounds arising from the statistically differing energies of each degree of freedom.

The way to explain this may be to start with the Planck-scale vacuum first articulated by Wheeler in his work on geometrodynamics [4], [5], conjectured to contain innumerable fluctuations which are themselves black holes, ultra-densely-packed with an average separation of $\lambda_{P}$, and establishing a collective event horizon with a Schwarzschild radius $r_{S P}=2 \hbar_{P}$ and thus diameter $4 \hbar_{P}$. From (1.3) this vacuum $(V)$ will emit its own blackbody spectrum at a temperature $k T_{V}=2\left\langle E_{V}\right\rangle=\hbar c^{3} / 8 \pi G M_{P}=E_{P} / 8 \pi=k \cdot 5.637 \times 10^{30} \mathrm{~K}$ and average $\left\langle E_{V}\right\rangle=E_{P} / 16 \pi$, with $\lambda_{\text {peak } V}$ calculated from Wein's (3.1) to be $\lambda_{\text {peak } V}=16 \pi^{2} \lambda_{P} / a$, and UV cutoff $\lambda_{\text {cutoff } V}=4 \lambda_{P}$ then calculated from (3.2). This is the very same as the collective event horizon diameter of the vacuum.

So, even when a photon with a statistically-high energy is emitted from a low-energy black hole fluctuation behind the horizon, that photon will be captured by one of the closely-neighboring higherenergy black hole fluctuations before it ever escapes the vacuum as a whole, because of the ultradense black hole packing behind the collective horizon. This capture by neighboring black holes enables us to envision how it is even possible to have $\left\langle E_{V}\right\rangle \lambda \geq \frac{1}{2} \hbar c$ based on (2.3) yield a fixed, general $\lambda_{\text {cutoff }}=\frac{1}{2} \hbar c /\left\langle E_{V}\right\rangle$ based on average energy, rather than having $E_{n V} \lambda_{n} \geq \frac{1}{2} \hbar c$ yield $\lambda_{n \text { cutoff }}=\frac{1}{2} \hbar c / E_{n V}$ with no particular general cutoff at all. Moreover, conversely, it is difficult to envision a physical process leading to $\langle E\rangle \lambda \geq \frac{1}{2} \hbar c$ from (2.3) based on an average energy, rather than $E_{n} \lambda_{n} \geq \frac{1}{2} \hbar c$ based on individual energies, unless this "neighborhood photon capture" arises from densely-packed black hole fluctuations in the vacuum. That is, the strong local nonlinear gravitational fields of densely-packed interacting black holes in the vacuum appear to be required to collectively capture photons based on an average energy $\langle E\rangle$ producing the "community cutoff" (3.2), rather than there being no particular cutoff due to the statistically-distributed energies $E_{n}$. 
The challenge, then, is to envision how this community cutoff carries over as predicted by (3.2) to a cutoff near $1 / 8$ of the Wein peak for ordinary blackbodies, when there is no evident involvement of densely-packed black holes. The only apparent explanation is that observing Planck-1901 blackbody radiation [3Error! Bookmark not defined.] must be physically synonymous with probing and observing Wheeler's gravitational vacuum over a spherical surface established by the radius $R=\hbar c / 4 \pi k T$ which is the equality in (2.2), which is identical in form to Hawking's (1.3), and which is much larger $R \gg r_{S P}=2 \lambda_{P}$ than the Schwarzschild radius of the vacuum. A simple analogy is a meter stick marked $1 / 8$ of the way from one end to the other: Viewing the stick from far away does not change the mark from being seen at its $1 / 8$ relative position even though everything about the stick scales down to look smaller.

Specifically, empty cavities used in blackbody experiments are simply experimental tools designed and constructed to eliminate vibrations of extraneous molecules, stray reflected light, and any other source of noise from inside the cavity, so we can observe how nature's own vacuum radiates in thermal equilibrium at any given temperature $T$. The equality in (2.2) written as $D=\hbar c / 2 \pi k T$ is therefore not anything intrinsic to the blackbody. It is set by the observational temperature with which the blackbody is probed, and simply tells us the spatial region of the vacuum being probed by this $T$. The greater the temperature, the smaller the region being probed. This is just like how the uncertainty limitation $\Delta r \geq \frac{1}{2} \hbar c / \mu$ from (2.4) tells us the microscopic spatial resolution obtained when we probe with energy $\mu$ in particle physics scattering experiments. In particle physics collisions, we use focused energies to scatter atoms or molecules or nuclei or nucleons and observe the particle debris. But there is a spatial resolution limit given by (2.4). When we apply a temperature $T$ to a blackbody and allow it to reach thermal equilibrium, we are scattering photons out of the vacuum and the observed debris is a blackbody spectrum. But here too there is a lower wavelength limit given by (3.2). Like the meter stick, no matter how "far away" the observational $T$ might be from $T_{V}=5.637 \times 10^{30} \mathrm{~K}$ of the vacuum, this UV cutoff is always observed at $\lambda_{\text {cutoff }} \cong \frac{1}{8} \lambda_{\text {peak }}$. And again, as reviewed following (3.2), this cutoff is a strictly-mathematical consequence of combining the Bekenstein bound (1.1) in the form of (2.2) with Wein's displacement law (3.1).

\section{Conclusion}

The derivation of the spatial measurement limitation (2.4) and the predicted UV cutoff (3.2) from the Bekenstein entropy bound (1.1), for a thermodynamic energy distribution specialized to a definite fixed probe energy, is strictly mathematical and non-speculative. So, if this predicted cutoff (3.2) for an ordinary blackbody is uncontradicted by the photon counting experiment quantified in (4.6), this would establish on observational in addition to mathematical grounds that the spatial measurement limitation (2.4) of the uncertainty principle is simply a special case of the statistical thermodynamics of the Bekenstein bound and the second law of thermodynamics. And, absent some compelling alternative explanation, this would provide empirical support for the physics of the cutoff (3.2) originating from the extreme black hole curvatures of the gravitational vacuum.

Specifically, if this cutoff is confirmed in ordinary blackbody radiation, there would appear to be no plausible explanation for this other than that when we observe this cutoff in an ordinary blackbody, we are really remotely observing the community cutoff of black hole fluctuations in the gravitational vacuum, analogously to the meter stick observed from afar. Theoretically, this would mean that the spatial measurement limitation of the uncertainty principle beyond which external observations cannot penetrate originates in the black hole event horizon of a gravitational vacuum beyond which external observations likewise cannot penetrate.

Consequently, experimental confirmation of this UV cutoff could help establish that the general theory of relativity which ab initio predicts black holes, provides a UV-complete, theoreticallyintelligible foundation not only for the entropic thermodynamics of the Bekenstein bound, but also for the uncertainty-based spatial resolution measurement limitation at the heart of quantum 
mechanics. Relatedly, because of Jacobsen's [15] derivation of the Einstein equation from the Bekenstein bound (1.1) and the laws of thermodynamics, coupled with the present derivation of the uncertainty principle relation (2.4) from the very same, such confirmation of the UV cutoff would indicate that the Bekenstein entropy bound and laws of statistical thermodynamics sit at the crossroads of general relativistic and quantum mechanical unification.

\section{References}

1. W. Heisenberg, About the descriptive content of quantum theoretical kinematics and mechanics, Zeitschrift für Physik (in German), 43 (3-4): 172-198 (1927)

2. M. Born and P. Jordan, To quantum mechanics, Zeitschrift für Physik. 34: 858 (1925)

3. M. Planck, On the Law of the Energy Distribution in the Normal Spectrum, Ann. Phys., 4, 553 (1901)

4. J. A. Wheeler, On the nature of quantum geometrodynamics, Annals of Physics, Volume 2, Issue 6, 604$614(1957)$

5. J. A. Wheeler, Geometrodynamics, Academic Press (1962)

6. J. D. Bekenstein, Universal upper bound on the entropy-to-energy ratio for bounded systems, Physical Review D. 23 (2): 287-298. (1981)

7. J. D. Bekenstein, Black Holes and the Second Law, Letters Nuovo Cimento 4, 737-740 (1972)

8. G. 't Hooft, Dimensional reduction in quantum gravity, https://arxiv.org/abs/gr-qc/9310026v1 (1993)

9. S. W. Hawking, Black hole explosions?, Nature. 248 (5443): 30-31 (1974)

10. R. M. Wald, General Relativity, University of Chicago Press (1984)

11. F. Halzen and A. D. Martin, Quarks and Leptons: An Introductory Course in Modern Particle Physics, Wiley (1984)

12. http://pdg.lbl.gov/2019/reviews/rpp2019-rev-phys-constants.pdf

13. H. P. Robertson, The Uncertainty Principle, Phys. Rev., 34 (1): 163-64 (1929)

14. L. Susskind, Black Holes, the Conservation of Information, and the Holographic Principle, https://www.cornell.edu/video/leonard-susskind-2-black-holes-conservation-of-information-holographicprinciple, see starting at about 32 minutes. (2014)

15. T. Jacobson, Thermodynamics of Spacetime: The Einstein Equation of State, Physical Review Letters. 75 (7): 1260-1263 (1995) 